Methods Intact female and male, or castrated and sham-castrated male BWF1 mice were used in this study. Cecal contents (microbiota) from different groups were transferred into female BWF1 mice shortly after weaning ( $\sim 26$ days $)$ and either disease or in vitro cell function (cell culture, flow cytometry) was evaluated. Feces were collected from adult mice and analyzed for either microbiota composition (deep sequencing of $16 \mathrm{~S}$ gene) or metabolomic profiles (mass spectrometry).

Results We have found that the composition of gut microbiota and metabolomic/lipidomic profiles differ between mature female and male BWF1 mice. Transfer of male microbiota to female BWF1 mice suppresses disease and increases survival. Further, we found that male microbiota may protect, in part, via an effect on tolerogenic $\mathrm{CD} 103^{+}$dendritic cells (CD103DC) that induce peripheral Tregs (pTregs) through TGF and retinoic acid (RA) production. Female BWF1 CD103DC have a decreased ability to induce pTregs and express retinaldehyde dehydrogenase, (RALDH2), an enzyme involved in RA synthesis. Transfer of male microbiota to female BWF1 mice reconstitutes both RALDH2 expression and the ability of the CD103DC to induce pTregs. Interestingly, castration of male mice significantly alters gut microbiota composition and metabolomic/lipidomic profiles by comparison to males, diminishes CD103DC function and decreases the ability of the microbiota to protect female mice from disease. The mechanisms underlying male microbiotamediated protection from disease are unknown, but may be mediated through the production of metabolites. We have identified several metabolites that are increased in male compared to both female and castrated male feces that function as retinoid $\mathrm{X}$ receptor agonists and enhance RALDH2 activity and increase pTregs in vivo.

Conclusions Our data suggest that androgens alter the composition and function of the gut microbiota in males, and the metabolites produced by the male microbiota may have potential for development into therapeutic strategies for the treatment of disease.

Acknowledgements Funded by Lupus Research Institute, Alliance for Lupus Research and National Institutes of Health.

\section{EF-06 ANTI-NUCLEAR ANTIBODIES (ANA) AND AIR POLLUTION: ULTRAFINE PARTICLES AND OZONE}

${ }^{1}$ Sasha Bernatsky*, ${ }^{2}$ Audrey Smargiassi, ${ }^{1}$ Shouao Wang, ${ }^{3}$ May Y Choi, ${ }^{1}$ Scott Weichenthal, ${ }^{4}$ Marianne Hatzopoulou, ${ }^{3}$ Marvin J Fritzler. ${ }^{1}$ McGill University, Montreal, QC, Canada; 2Université de Montreal, Montreal, QC, Canada; ${ }^{3}$ University of Calgary, Calgary, AB, Canada; ${ }^{4}$ University of Toronto, Toronto, ON, Canada

\subsection{6/lupus-2018-Ism.86}

Background Previous studies suggest links between air pollution (i.e. fine particulate matter, PM2.5) and serum antibodies related to rheumatic diseases. No one has yet examined associations between anti-nuclear antibodies (ANA) and ultrafine particles (UFP) or ozone (O3), both of which can enter through the lungs and may have the potential to trigger systemic effects.

Methods Our analyses were based within the CARTaGENE general population cohort $(n=20,000)$ based in the province of Quebec, Canada. We determined baseline ANA (HEp-2000, Immuno Concepts) on a random sample. Air pollutant exposures were assigned by linking subjects' residential postal codes with estimated levels (determined by hybrid approaches including satellite imagery and modelling). We performed multivariable logistic regression models for the outcome of positive ANA, assessing for independent effects of UFP (available for Montreal only) and $\mathrm{O} 3$ in separate models, adjusting for age, sex, smoking, and self-reported ancestry.

Results ANA positivity of at least 1:160 occurred in 713 (20\%) of 3578 randomly selected patients tested. The ANA positive subjects were more likely than ANA negative subjects to be female $(63 \%$ vs $49 \%)$ while the average age (55.4 vs $54.0)$ and percent never-smokers (37\% vs $40 \%)$ were similar. There was missing information on covariates for 232 subjects, which therefore were not included in the model estimates.

There was a trend for higher average UFP: exposure in ANA positive subjects (24 606 particles $/ \mathrm{cm}^{3}$, standard deviation, SD 4979) versus ANA negative (24328, SD 5078), while average ozone levels were very similar $(22.5$ vs $22.6 \mu \mathrm{g} / \mathrm{m})$. The multivariable model (table 1) for UFP showed a trend to higher levels in the ANA positive group $(1.008,95 \% \mathrm{CI}$ 0.982 to 1.034$)$ while in the multivariable model for $\mathrm{O} 3$ the OR was very close to the null value $(0.996$, 95\% CI 0.965 to 1.029). In all models, risk factors for ANA positivity included older age and female sex, with trends for lower ANA positivity in French Canadians.

Conclusions We saw a non-significant trend towards higher UFP levels in ANA positive versus negative subjects, while O3 levels seemed very similar in the two groups. Expected trends for more ANA positivity with older age and female sex was seen. Further study of UFP levels with a larger sample size is in progress. If confirmed, these results may strengthen the hypothesis that air pollution is an environmental trigger of immune system activation.

\begin{tabular}{|c|c|c|c|}
\hline $\begin{array}{l}\text { Single pollutant model, Ozone, } \\
\mathrm{n}=3346\end{array}$ & Adjusted odds ratio & $95 \%$ & $\mathrm{Cl}$ \\
\hline $03\left(\mu \mathrm{g} / \mathrm{m}^{3}\right)$ & 0.996 & 0.965 & 1.029 \\
\hline Age (continuous) & 1.024 & 1.013 & 1.036 \\
\hline Female & 1.778 & 1.492 & 2.118 \\
\hline Current (versus never or past) smoker & 1.038 & 0.833 & 1.294 \\
\hline French Canadian Caucasian & 0.843 & 0.703 & 1.012 \\
\hline Montreal & 0.980 & 0.793 & 1.211 \\
\hline Ultrafine particules, $n=1371$ & Adjusted Odds & $95 \%$ & $\mathrm{Cl}$ \\
\hline (Montreal) & Ratio & & \\
\hline UFP (1000 particles $\left./ \mathrm{cm}^{3}\right)$ & 1.008 & 0.982 & 1.034 \\
\hline Age (continuous) & 1.028 & 1.011 & 1.045 \\
\hline Female & 2.054 & 1.558 & 2.708 \\
\hline Current (versus never or past) smoker & 1.119 & 0.802 & 1.561 \\
\hline French Canadian Caucasian & 0.749 & 0.571 & 0.982 \\
\hline Montreal & NA & NA & NA \\
\hline
\end{tabular}

Acknowledgements This research was supported by the CIHR. CARTaGENE is a member of the Canadian Project for Tomorrow cohort. 